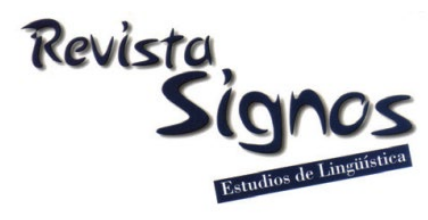

\title{
Incongruencia epistémica, partículas intensificadoras y elaboración de la información ${ }^{1}$
}

\section{Epistemic incongruence, intensifying particles and information elaboration}

\author{
Amparo García-Ramón \\ Grupo VAL.Es.Co, Universitat de VALÈnCia \\ ESPAÑA \\ amparo.garcia-ramon@uv.es
}

\section{Recibido: 30-IX-2017 / Aceptado: 21-XI-2018}

DOI: $10.4067 /$ S0718-09342019000300804

\section{Resumen}

En el presente estudio se describe un patrón discursivo hallado en intervenciones reactivas tras aserciones. El patrón contiene una partícula intensificadora del acuerdo (p. ej., 'claro', 'por supuesto', 'evidentemente') seguida de un segmento en el que se elabora la información propuesta en la aserción iniciativa. Se argumenta que, en los contextos analizados, las partículas señalan la existencia de una incongruencia epistémica: aunque el segundo hablante muestra acuerdo con el contenido semántico propuesto por el primero, dicho contenido resulta conocido e incluso obvio para el segundo hablante y la aserción es, en ese sentido, problemática. A partir del análisis, se propone una distinción entre dos niveles dentro del concepto general de (des)acuerdo: un nivel en el plano del contenido y otro nivel relacionado con las asunciones sobre la configuración epistémica que propician la ejecución misma de un acto de habla asertivo.

Palabras Clave: Interacción, epistemicidad, (des)acuerdo, incongruencia, partículas intensificadoras. 


\begin{abstract}
This paper explores a discourse pattern found in responses to assertions. The pattern includes a particle that stresses agreement (e.g., Sp. claro, por supuesto, evidentemente) followed by a segment where the second speaker elaborates on the information provided by the first. It is argued that, in the contexts analysed here, these particles index epistemic incongruence: although the second speaker shows agreement with the semantic content proposed by the first, the content is known or even obvious for the second speaker and the assertion is therefore, in a sense, problematic. Drawing from this analysis, a distinction is proposed between two levels within the general category of (dis)agreement: one level relates to content and the other relates to the assumptions about the epistemic configuration that are carried in an assertive speech act itself.
\end{abstract}

Key Words: Interaction, epistemics, incongruence, (dis)agreement, intensifying particles.

\title{
INTRODUCCIÓN
}

En las últimas décadas, se ha consolidado una línea de investigación dentro del análisis conversacional (AC) centrado en la gestión del conocimiento en interacción (ver, entre otros, Heritage \& Raymond, 2005; Stivers, 2005, 2011; Clift, 2006, 2007; Stivers, Mondada \& Steensig, 2011; Sidnell, 2012; Heritage, 2012, 2013a, 2013b). Según estos estudios (en adelante, estudios de epistemicidad en interacción o EEI), existe una serie de esquemas básicos que se activan intersubjetivamente cuando se ejecutan movimientos conversacionales susceptibles de ser entendidos en términos de epistemicidad. Por ejemplo, preguntar conlleva el presupuesto de que el hablante carece de una información de la que dispone el oyente, mientras que afirmar conlleva el presupuesto contrario. Este esquema se activa por defecto, pero los hablantes tienen a su disposición una serie de estrategias conversacionales con las cuales pueden neutralizarlo como, por ejemplo, atenuar las primeras partes e intensificar las segundas. Algunos de los mecanismos que se han descrito como representativos de este tipo de modalización en inglés son, para las primeras partes, las formas evidenciales (it sounds, it seems), los verbos de opinión (I think) y los apéndices confirmatorios (isn't it?) (Heritage \& Raymond, 2005; Sidnell, 2012) y, para las segundas, las partículas intensificadoras del acuerdo (of course) (Stivers, 2011), las repeticiones modificadas (Stivers, 2005), las autocitas (Clift, 2006, 2007) o, de nuevo, los apéndices confirmatorios, esta vez en posición reactiva (Heritage \& Raymond, 2005). En ocasiones, se producen incongruencias epistémicas, es decir, desajustes entre la interpretación que dos hablantes hacen de la situación en términos epistémicos (Stivers et al., 2011).

A pesar de la abundante producción científica generada por los EEI en el ámbito anglosajón, todavía son escasos los estudios para el español que asumen esta perspectiva de análisis (Cornillie \& Gras, 2015; Vázquez Carranza, 2009, 2017; GarcíaRamón, 2018a, 2018b). El objetivo del presente trabajo es describir un patrón 
discursivo recurrente en el que las partículas asertivas intensificadoras del acuerdo ('claro', 'desde luego', 'efectivamente', 'en efecto', 'evidentemente', 'naturalmente', 'por supuesto', 'sin duda') actualizan significados secundarios los cuales, se argumenta, están relacionados con la señalización de incongruencias epistémicas. Se adopta la definición de Taranilla (2016), inspirada en la de Couper-Kuhlen y Thompson (2000, 2008), según la cual un patrón discursivo es:

"aquella práctica recurrente en la configuración del discurso que, sin llegar a sedimentarse en una forma fija, constituye una rutina habitual a la hora de disponer los materiales informativos que configuran una producción textual, así como las relaciones entre estos" (Taranilla, 2016: 260).

El patrón descrito en este trabajo aparece en respuestas a aserciones y se compone de una muestra de acuerdo y un segmento en el cual se expone una elaboración de la información sobre la cual se ha mostrado acuerdo. La aserción inicial es producida por un hablante (H1) y la muestra de acuerdo, junto con la elaboración de la información, es producida por un segundo hablante (H2). El patrón dialogal completo podría formularse como $<$ [aserción $]_{\mathrm{h} 1}+$ [acuerdo + elaboración de la información $]_{\mathrm{h} 2}>$. Nos centramos solamente en la intervención de H2: <acuerdo + elaboración de la información $>$. Por tanto, se analizan exclusivamente intervenciones reactivas (aquellas que responden a intervenciones anteriores) o reactivo-iniciativas (aquellas que, por una parte, responden a intervenciones anteriores y, por otra, provocan una respuesta en intervenciones posteriores) (Briz \& Grupo Val.Es.Co., 2003; Grupo Val.Es.Co., 2014). Analizamos solamente casos en los que el componente <acuerdo> se materializa en alguna de las partículas mencionadas.

El corpus contiene 14 interacciones en diversos géneros (conversaciones coloquiales, entrevistas periodísticas y entrevistas sociolingüísticas) en los cuales existen diferentes tipos de roles sociofuncionales los cuales, potencialmente, inciden en la adopción de diferentes posicionamientos epistémicos. Las conversaciones han sido tomadas del corpus Val.Es.Co. 2.0 (Cabedo \& Pons, en línea), que se caracteriza por el elevado grado de espontaneidad de las muestras. Las entrevistas periodísticas, compiladas para los fines de una investigación en curso (García-Ramón, 2018c) se dividen entre aquellas en las que el entrevistado es un político en activo y aquellas en las que el entrevistado es un experto en algún tema concreto. Por último, las entrevistas sociolingüísticas forman parte del proyecto (PRESEEA, en línea).

\section{Marco teórico}

\subsection{El componente social en los EEI: la noción de incongruencia epistémica}

El análisis según el cual la producción de una aserción descansa sobre la base de ciertas condiciones subyacentes no es, en realidad, demasiado novedoso: se puede 
reconocer su origen en la teoría de los actos de habla -concretamente, a la condición preparatoria de los actos asertivos (no es obvio para el hablante y el oyente que el oyente sabe y no necesita que le recuerden la proposición 'p' (Searle, 1969)) - o, en términos del AC, en la regla conversacional según la cual no se debe contar al interlocutor lo que ya sabe (Sacks, 1992). La novedad de los EEI es la importancia otorgada a cuestiones sociales en la transmisión de información: los esquemas epistémicos básicos tienen implicaciones sobre quién tiene el 'derecho' o la 'autoridad' para conocer y expresar diferentes tipos de contenidos frente a unos interlocutores determinados.

Dentro de los EEI, uno de los elementos que se asume como prueba de la relevancia de la gestión de cuestiones epistémicas para los propios interlocutores viene precisamente de los casos en que existe algún tipo de problematicidad o negociación en torno a los esquemas epistémicos básicos asociados con un acto de habla concreto: nos interesan aquí los actos de tipo asertivo, dentro de las que incluimos tanto acciones puramente informativas, como una narración, como acciones en las que se añade al contenido factual un contenido de tipo evaluativo ${ }^{2}$.

La idea de problematicidad, sugerida en los diferentes estudios mediante el uso de nociones como 'resistencia' (Shaw \& Hepburn, 2013; Vázquez Carranza, 2016) o 'competición' (Stivers, 2005), ha sido descrita de manera más sistemática por Stivers et al. (2011) dentro de la categoría general 'incongruencia epistémica'. Dentro de esta categoría general, los autores distinguen entre casos de incongruencia de acceso epistémico' -cuando los interlocutores manifiestan no tener la misma percepción sobre qué sabe cada uno de ellos- e 'incongruencia de primacía epistémica' -cuando los interlocutores manifiestan no tener la misma percepción sobre quién tiene derechos primarios o autoridad epistémica sobre un referente concreto-. Por ejemplo, según Stivers et al. (2011: 11), en (1) existe 'incongruencia de acceso epistémico’: Tara predice mal los conocimientos de su oyente $y$, por tanto, narra un hecho partiendo de la base de que es novedoso para su interlocutora (línea 1). En el turno siguiente (línea 3), Kris repara el error:

(1)

1 Tara: My mom left me this whọ:le long message on my cell phone last night

2 about thuh directions,

3 Kris: Yea(h)h I kn(h)ow, I was there.

(Stivers et al., 2011, 11)

En el ejemplo (2), extraído de Clift (2006), J y V son dos mujeres que están hablando de la hija de $\mathrm{V}$ y la familia de aquella. En este contexto, J propone una evaluación de la hija de $\mathrm{V}$ y su familia, atenuada mediante el apéndice confirmatorio (aren't they, línea 1). V valida la contribución de J de manera rotunda (línea 2) y J vuelve a proponer una opinión. Lejos de ofrecer una simple muestra de acuerdo, V reclama 
que ella misma también poseía esa visión del asunto antes de este momento (prueba de ello es que $\mathrm{V}$ dijo exactamente lo mismo a su yerno en un momento anterior a esta interacción) y que, además, tiene derechos epistémicos primarios sobre este referente, que está más cerca de su experiencia vivencial que de la de su interlocutora.

(2)

$1 \mathrm{~J}$ : They're a lovely family now aren't they

$2 \mathrm{~V}: \mathrm{Mm}$ : They are:ye[s

$3 \mathrm{~J}:$ eeYes

$4 \mathrm{~V}$ : Yes

$5 \mathrm{~J}: \mathrm{Mm}$ : All they need now is a little girl to complete i:t.

$6 \mathrm{~J}:$ heh heh

7 V: Well I said tuh Jean how about it so our Bill (0.2) laughingly said 'ey she'll havetuh ask me

8 first now

$9 \mathrm{~J}:$ h:ha $: \underline{\text { ha: }}$

(Clift, 2006, 130)

\subsection{Roles sociofuncionales en el contexto interactivo general y en el contexto interactivo concreto}

Los roles sociofuncionales, íntimamente relacionados con cuestiones de autoridad y subordinación epistémica, se relacionan con el contexto interaccional en dos niveles diferentes. Se parte para el establecimiento de estos dos niveles de la distinción propuesta por Briz y Albelda (2013) entre 'contexto interactivo general' (CIG) y 'contexto interactivo concreto' (CIC). En el primer nivel (el CIG), se incluyen aquellos rasgos del contexto que están en gran medida predeterminados por el molde general de la interacción y que pueden considerarse, por tanto, rasgos del género en un sentido estático (para una discusión de lo estático y lo dinámico dentro de la variación diafásica, ver Briz 2010a, 2010b, 2012). Por ejemplo, en el caso de las entrevistas, en un sentido general, uno de los hablantes cumple el rol de entrevistador y otro hablante cumple el rol de entrevistado. En el segundo nivel (el CIC), se incluyen los rasgos que pertenecen a secuencias discursivas particulares y que, por tanto, pueden variar a lo largo de la interacción (por ejemplo, en una secuencia de relato dentro de una conversación coloquial, los roles más efímeros de narrador y oyente). Por tanto, "la noción de contexto interaccional concreto dinamiza (...) el concepto más general de situación de comunicación (...) y explica la actividad lingüística en una contextualización seriada, contexto preciso a contexto preciso" (Briz \& Albelda, 2013: 300).

Así, dentro del CIG encontramos roles relativamente estables, mientras que, si se considera cada CIC, se observa que emergen roles con carácter dinámico, que se negocian y se establecen secuencia a secuencia. Para los objetivos de este trabajo, 
interesan específicamente aquellos aspectos del rol sociofuncional que tienen que ver con el diferente crédito socioepistémico otorgado a cada uno de los participantes. Este tipo de rol epistémico puede hallarse en el nivel del CIG - cuando, como en el caso de las entrevistas a expertos, uno de los participantes es tratado como el hablante epistémicamente más autorizado a lo largo de toda una interacción- o en nivel del CIC - cuando el crédito socioepistémico otorgado a cada participante depende de cuál sea el referente comunicativo sobre el que se habla en una secuencia particular y cuál sea la relación de cada interlocutor con dicho referente-

\subsection{Las partículas modales asertivas como marcas de obviedad o redundancia}

Las partículas como 'claro', 'desde luego', 'efectivamente', 'en efecto', 'evidentemente', 'naturalmente', 'por supuesto' o 'sin duda' son elementos afirmativos de polaridad máximamente positiva: conforman un paradigma dentro del cual el 'archipragmema' es la simple afirmación ('sí) (Martín Zorraquino, 1994). A diferencia de esa simple afirmación, estas partículas añaden un contenido modal que puede responder a diferentes propósitos comunicativos. Se trata de palabras no lexemáticas que, como las interjecciones, son equivalentes de oración (Martín Zorraquino, 1994) y pueden, así, funcionar como actos de habla completos. Para el presente trabajo, nos interesan solamente aquellos usos dialogales en los que las partículas mencionadas se emplean para mostrar acuerdo con una aserción previa producida por otro hablante. Mediante el uso de una de las partículas, H2 recoge la información contenida en la aserción producida por $\mathrm{H} 1 \mathrm{y}$, al mismo tiempo, intensifica dicha aserción ${ }^{3}$.

Algunos trabajos han sugerido que estas partículas no solamente muestran acuerdo con una afirmación o refuerzan la seguridad, más específicamente, señalan que esa información es compartida por todos los interlocutores, de dominio general o, en una palabra, evidente. Así, Martín Zorraquino y Portolés (1999) sugieren que una de las funciones de claro es señalar un miembro como repetido o redundante. Para Sánchez Jiménez (2017), naturalmente' 'indica que el miembro del discurso al que afecta ha de interpretarse como una consecuencia esperada a partir de la información previa que, al respecto, tiene el hablante'. También Fuentes (2009: 225) propone que 'naturalmente' se refiere a un contenido "conocido por la comunidad, evidente, deducido de forma natural de los hechos". Por su parte, Briz (2016: 112) señala que mediante el uso de estas partículas el hablante se apoya en la lógica, en lo que parece ser compartido o incluso es "percibido como evidente" y, a través de ello, "refuerza argumentativamente lo expresado" lo cual, en los usos dialogales que nos interesan aquí, equivale a reforzar el acuerdo.

Ahora bien, aunque las partículas funcionan como reforzadoras del acuerdo, el valor de evidencia, de obviedad o de redundancia puede constituir, en otro sentido, un prerrequisito para la problematización de la aserción previa. Así lo sugiere, por 
ejemplo, Maldonado (2010: 89). El autor distingue, entre otras funciones, una función correctiva de 'claro' en la cual "el paso que anuncia la corrección es la marcación de una afirmación como obvia": la información sobre la cual tiene ámbito la partícula se evalúa como insuficiente o se apunta a que el ángulo para su presentación no es el más apropiado. La noción de incongruencia que exploramos en este trabajo se alinea en gran medida con este apunte de Maldonado.

\section{Corpus y método}

El estudio se basa en el análisis cualitativo de un corpus de 4 conversaciones coloquiales (Cabedo \& Pons, en línea), 4 entrevistas periodísticas a políticos en activo (entrevistas políticas) (García-Ramón, 2018c), 3 entrevistas periodísticas a expertos en diferentes temas (entrevistas divulgativas) (García-Ramón, 2019) y 3 entrevistas sociolingüísticas (PRESEEA, en línea). Las conversaciones se caracterizan por tener el grado máximo de espontaneidad, mientras que en las entrevistas se dan fines transaccionales que limitan dicha espontaneidad: en las entrevistas periodísticas, los fines transaccionales tienen que ver con el descubrimiento de contenidos sobre los entrevistados; en las sociolingüísticas, con la producción de determinadas formas lingüísticas y tipos discursivos a partir del empleo de cuestionarios relativamente preestablecidos.

Al seleccionar materiales de naturaleza distinta, se persigue mostrar cómo los roles sociofuncionales, especialmente en lo que a la autoridad epistémica se refiere, se configuran de forma diferente en los diferentes contextos interactivos generales (CIG) (ver sección 1.2). Así, no se dan en las conversaciones asimetrías preestablecidas, aunque sí se pueden construir dichas asimetrías en cada contexto interactivo concreto (CIC). En cambio, en todas las entrevistas se dan roles sociofuncionales dependientes estrictamente del molde del género y, por tanto, del CIG (esto es, los roles de entrevistador y entrevistado). No obstante, las diferentes entrevistas no se entienden como un conjunto homogéneo sino que, a pesar de las similitudes relacionadas con los roles de entrevistador y entrevistado, existen otros roles de naturaleza epistémica que varían según el tipo de entrevista: así, por ejemplo, los entrevistados de las entrevistas divulgativas se consideran, en principio, como hablantes epistémicamente más autorizados que los entrevistadores, mientras que lo contrario sucede en las entrevistas políticas. Por último, en las entrevistas sociolingüísticas, las estrategias interaccionales relacionadas con el propósito general del género (lograr obtener material lingüístico producido por el informante) hacen que los roles socioepistémicos se desdibujen en sentidos que se observarán en el análisis.

El estudio tiene su punto de partida en los trabajos sobre epistemicidad del AC etnomedológico, en los que se argumenta que la concentración de mecanismos de intensificación en las segundas partes de secuencias de aserción-acuerdo tiene que ver con el equilibramiento del posicionamiento epistémico en interacción. A partir de las 
ideas presentadas en dichos trabajos, se han seleccionado para el análisis las partículas modales asertivas en posición reactiva, puesto que, según la bibliografía hispánica (ver sección 1.3) una de las funciones principales de dichas partículas es intensificar el acuerdo. Por tanto, se han seleccionado, de las 14 interacciones mencionadas más arriba, todas las secuencias en que las partículas modales asertivas aparecen en posición inicial o hacia el inicio en turnos reactivos en los que se expresa acuerdo.

A continuación, se ha procedido a la observación de los entornos interaccionales en los que aparecen dichas partículas, para lo cual se ha empleado el aparato metodológico propio del AC, de carácter eminentemente inductivo, que incluye canónicamente las siguientes fases: (a) detectar un fenómeno o una forma candidata a ser considerada fenómeno (b) elaborar una colección del fenómeno y establecer sus límites formales, (c) identificar las regularidades interaccionales en la interacción sobre la base de la posición y composición de las acciones, teniendo en cuenta sobre todo las orientaciones de los participantes hacia ellas, (d) dividir los casos en subcolecciones teniendo en cuenta sus características interaccionales relevantes (para una explicación más detallada de los métodos del AC, ver, p. ej., ten Have, 1999; Hutchby \& Wooffitt, 2008; Sidnell, 2010, 2013).

La observación de la colección de casos en los que se responde a una aserción mediante una de las partículas mencionadas ha llevado a detectar la existencia de un patrón discursivo habitual (el patrón < acuerdo + elaboración de la información>, el cual se da en casi la mitad de los casos de los usos reactivos de las partículas) (fase a). Posteriormente (fase b, sección 3.1), se ha analizado con mayor detenimiento dicho patrón para observar sus límites formales. Finalmente (fases c y d, sección 3.2), se han analizado las orientaciones de los participantes en interacción y las diferentes funciones interaccionales, que constituyen subgrupos dentro del fenómeno general.

\section{El patrón discursivo <acuerdo + elaboración de la información>}

Se han encontrado 86 casos en los que una partícula intensificadora se sitúa en posición inicial o hacia el inicio de intervención con la función primaria de mostrar acuerdo con una aserción previa. En 40 de ellos, la partícula se inserta dentro del patrón < acuerdo + elaboración de la información>, por lo que se entiende que dicho patrón es un entorno interaccional habitual para el uso de partículas modales asertivas. A pesar de la clara predominancia de la partícula 'claro', se han querido mantener los ejemplos con las demás partículas intensificadoras porque el objetivo del estudio es describir el patrón discursivo completo, más allá de las formas lingüísticas particulares que se empleen en cada caso.

En los siguientes apartados, describimos los subtipos formales (3.1.) y las funciones interaccionales (3.2.) del patrón interaccional mencionado. 
Argumentaremos que, cuando aparecen insertas en este patrón, las partículas señalan que la aserción anterior es cierta o plausible en lo que respecta a su contenido, pero problemática en términos de su configuración epistémica básica, puesto que el contenido expresado por $\mathrm{H} 1$ es obvio o esperable desde el punto de vista de $\mathrm{H} 2$. Se trata, por tanto, de marcadores que, en estos contextos, adquieren instrucciones de procesamiento diferentes de su significado básico, las cuales están asociadas con la existencia de incongruencias epistémicas.

Por tanto, proponemos un análisis en dos niveles diferentes dentro del concepto general de (des)acuerdo: un nivel que se desarrolla en el plano del contenido (es decir, el (des)acuerdo con la sustancia semántica) y otro nivel en el plano de las condiciones preparatorias (es decir, el (des)acuerdo con las asunciones sobre la configuración epistémica que propician la ejecución del acto de habla en sî), en el cual se desarrolla la incongruencia epistémica ${ }^{4}$. Ambas dimensiones se entrecruzan, de forma que en contextos en que la respuesta a una aserción es una muestra de acuerdo (cuando los dos hablantes proponen la plausibilidad de un mismo contenido) puede existir incongruencia (cuando los hablantes negocian el esquema epistémico básico) (Figura $1)$.

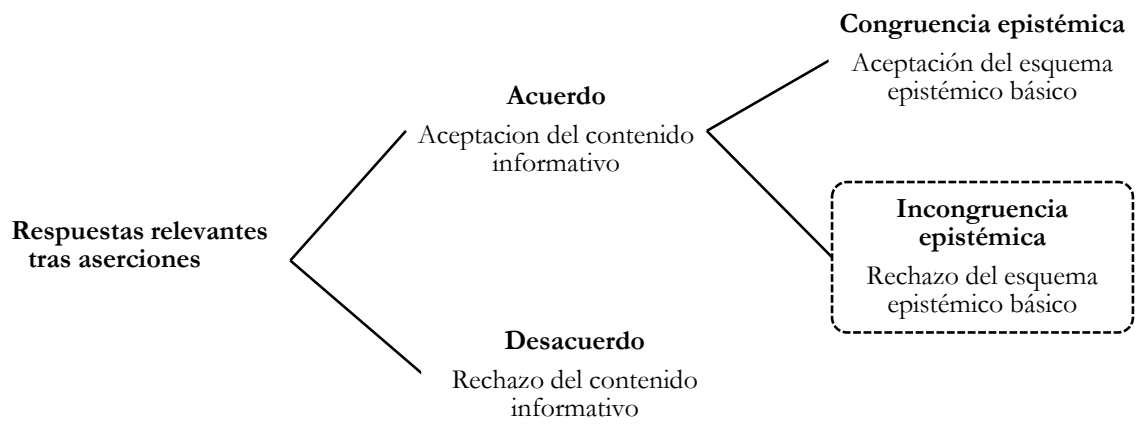

Figura 1. Respuestas relevantes tras actos de habla asertivos: (des)acuerdo e (in)congruencia.

\subsection{Subtipos del componente <elaboración de la información>}

Los cuatro modos de elaboración de la información propuestos a continuación tienen en común que, mediante su uso, $\mathrm{H} 2$ muestra que su conocimiento del tema trasciende las pretensiones de la afirmación propuesta por H1. Frente a la visión más acotada propuesta en la aserción previa, H2 ofrece una visión panorámica de la cuestión tratada. En nuestro corpus, la estrategia de elaboración de la información adopta al menos cuatro formas básicas: a) generalización del análisis, b) señalamiento de la causa, c) aportación de nuevos datos y d) desarrollo cronológico o causal. 
a) Generalización del análisis: $\mathrm{H} 2$ propone un sintagma nominal que equivale al tema de la interacción, el cual, hasta el momento, permanecía implícito (p. ej., la sociedad, la educación, el sistema) y añade un predicado a ese sintagma. El resultado es una declaración categórica, que pretende ser representativa en un sentido más amplio del que propone H1 con su afirmación (ejemplos 3-4).

(3)

1 RM: ¿y las puertas giratorias?

2 PS: se acabaron hay que cerrarlas

3 RM: mira Fe- Felipe González

4 PS: sí claro es que la sociedad ev- evoluCIOna/ y hoy Felipe González si DEJAra el

5 gobierno estoy convencido (Entrevista de Risto Mejide a Pedro Sánchez en Viajando con Chester, Cuatro)

(4)

$1 \mathrm{JE}$ : o sea diciendo esto / a pesar de que ahora puedan venir unos anuncioos / n- donde

2 pueda haber una entidad bancaria $\uparrow /$ bueeno / forma parte del sistema

3 JLS: ¡CLAro! ¡forma parte del sistema!

$4 \mathrm{JE}:{ }^{\circ}(\text { bueno })^{\circ}$

5 JLS: ¡el sistema es muy listo!

(Entrevista de Jordi Évole a José Luis Sampedro en Salvados, La Sexta)

b) Señalamiento de la causa: En un sentido similar, H1 puede mostrar acuerdo con una aserción producida por $\mathrm{H} 2$ y, además, aportar la causa que ha dado lugar al estado de cosas representado en esa aserción (la cual, en algunos casos, puede presentarse como argumento). De esa forma, se establece una lógica según la cual se infiere que $\mathrm{H} 2$ ya poseía esa información de manera consolidada y (más) completa; dentro de esa información más abarcadora, la información propuesta por H1 constituye solamente una parte. Es frecuente introducir esta explicación mediante la conjunción causal porque ${ }^{5}$.

(5)

$1 \mathrm{C}: \quad$ era la Resurrección a las doce de la noche $\uparrow / \mathrm{y}$ las chiquillas casi sin venir

2 ((diciendo)) digo mira $\uparrow$ si yo no tengo muchas ganas de ir a la Resurrección/ y es

3 que hacía UN FRÍO que pelaba y no me llevé abrigo/ me llevé ((na(da) más)) que

$4 \quad$ el traje de chaqueta 
5 P: $\quad$ Siclaro! porque no te esperas $\uparrow /$ pero noo/ cuando te vas a

6 esos sitios [aunque $\mathrm{NO}$ lo $(($ uses $))=$ ]

(Corpus Val.Es.Co. 2.0, Conversación 0030)

c) Aportación de nuevos datos: En tercer lugar, la elaboración de la información se puede dar en forma de añadidura de nuevos datos o segmentos informativos, frecuentemente introducidos por la conjunción $y$, que complementan lo dicho por H1, como en (6) (sobre usos como el de (6), en los que, además de señalar acuerdo, un hablante recupera el tuno, ver 3.2.2).

(6)

$1 \mathrm{~L}: \quad$ pero obviamente/ ella tampoco/ que no/ [o sea]

$2 \mathrm{E}$ : [no no]/ no lo dice para encabronarte/ si

3 es verdad $\uparrow / \operatorname{simplemente}(())$

4 L: ¡claro!/ ni y y y/ si yo le dijera/ oye mira/ no voy al cine/ ¿sabes?/ supongo que

5 se sentiría/ súper violentada/ ella también/ ¿sabes?// espero/ (RISAS)

$6 \mathrm{E}: \quad(\mathrm{RISAS}) \mathrm{S}$

(Corpus Val.Es.Co. 2.0, Conversación 0039)

d) Desarrollo cronológico o causal. Finalmente, existe una manifestación del miembro <elaboración de la información> en que H2 aporta un desarrollo de tipo cronológico (explica qué sucedería tras lo expuesto por H1) o causal (explica cuál sería la consecuencia de lo expuesto por H1). Con frecuencia este segmento aparece introducido por la conjunción $y$, en ocasiones seguida del adverbio entonces, como en (7) (de nuevo, sobre la función de reclamar el turno y su compatibilidad son otras funciones propuestas, ver 3.2.2).

(7)

1 JAM: cuando ves ese tipo de cosas $\rightarrow$

2 RM: sí/ se arrepiente de haber entrado en eel

3 JAM: claro/ y entonces/ te das cuenta o/ hmm para no favorecer cuando empiezas

4 tú/ mira/ y nadie lo ha buscao/ pero aparecen así// todo el discurso amoroso es

5 un discurso/ que engaña mucho

(Entrevista de Risto Mejide a José Antonio Marina en Viajando con Chester, Cuatro)

\subsection{Funciones interaccionales del patrón <acuerdo + elaboración de la información>}

A partir de una respuesta formada por los componentes <acuerdo $>$ y $<$ elaboración de la información>, H2 reclama acceso epistémico previo y, en algunas 
ocasiones, además, reclama primacía epistémica sobre el referente. En ambos casos, se trata de momentos interaccionales en que se indica que existe incongruencia epistémica: es decir, el hablante H2 manifiesta implícitamente que la interpretación que ha hecho $\mathrm{H} 1$ de la situación en términos de su configuración epistémica básica necesita una revisión o corrección de algún tipo. Ello no quiere decir, como se comprobará a lo largo del análisis, que se trate necesariamente de situaciones confrontacionales: hay motivaciones de naturaleza afiliativa por las cuales un hablante puede señalar que existe incongruencia epistémica ${ }^{6}$. En el corpus, la necesidad de manifestar esta instrucción metadiscursiva obedece esencialmente a tres metas interaccionales: (i) estrechar los lazos con H1 (3.2.1.), (ii) reclamar primacía epistémica (3.2.2.), y (iii) deslegitimar acciones conversacionales potencialmente amenazantes (3.2.3.).

\subsubsection{Reforzar el acuerdo y estrechar los lazos}

La primera de las funciones mencionadas es de carácter socializador. En el ya clásico estudio de Pomerantz (1984) sobre secuencias de acuerdo, la autora propone que, puesto que existe una preferencia general por el acuerdo en la interacción espontánea, en secuencias de acuerdo, H1 puede interpretar que el acuerdo manifestado por $\mathrm{H} 2$ es fruto de una mera inercia conversacional. Por tanto, los hablantes tienden a intensificar las segundas partes para expresar que la muestra de acuerdo obedece a una verdadera coincidencia de visiones. Una de las formas de señalar esto es mostrar que, para H2, la opinión en juego es anterior al momento de la enunciación.

En (8), extraído de una entrevista sociolingüística del PRESEEA, la entrevistadora (E) está tratando de animar a la informante (I) para que narre acontecimientos de su infancia. En las entrevistas del PRESEEA, en general, hay un interés por parte del/de la entrevistador/a de que el/la informante produzca fragmentos lingüísticos con la mayor relajación posible y, fruto de este interés, son constantes las contribuciones de carácter colaborativo y valorizante por parte de E. Esta intención es especialmente visible en este fragmento, en que I se ha mostrado dubitativa sobre el interés de su historia, por lo que es esperable que E se muestre especialmente complaciente. Una manera de animar a I es manifestar que los datos no solo 'interesan' a los investigadores, sino que les 'encantan' (línea 4). Una segunda manera de hacerlo es mostrar identificación con la historia de I. Ante la narración de I, centrada en la anécdota personal, E muestra acuerdo ('claro') y elabora sobre esa información. E generaliza el fenómeno ('en aquella época', línea 7). A continuación, completa la historia de I al anticipar con 'nada nada' (línea 11) la escena que I se dispone a describir. Finalmente, hace explícita la identificación ('eso también yo lo he vivido ya', línea 13). Mediante estos recursos, E muestra que no solamente está de acuerdo con la 
descripción de I, sino que ella misma ya había percibido esa situación con anterioridad.

1 I: ¿Oos interesan $<$ simultáneo $>$ estos datos? $</$ simultáneo $>$

$2 \mathrm{E}: \quad<$ simultáneo $>$ sí sí sí $</$ simultáneo $>$

$3 \mathrm{I}: \quad$ porque a lo mejor es como una $<$ simultáneo $>$ fotonovela $</$ simultáneo $>$

$4 \mathrm{E}$ : $<$ simultáneo $>$ nos interesan $</$ simultáneo $>$ y nos encantan

$5 \mathrm{I}$ : pues yo te cuento entonces // entonces $\mathrm{mm}<$ vacilación/ $>$ mi hermano y yo estábamos

6 porque se veraneaba $<$ alargamiento $/>$ no se veraneaba

7 E: claro// en aquella época

8 I: se iba a casa de los abuelos hombre sí / veraneaba la gente a San Sebastián / y tenían la

9 caseta/ y les arrimaban la caseta a la playa / y se metían los señores con los sombreros / y

10 luego les ponían la toalla pero los que no teníamos dinero < alargamiento/>

\section{$11 \mathrm{E}:$ nada/ nada}

12 I: no nos comíamos una rosca <risas $=$ "I" $/>$

$13 \mathrm{E}$ : eso también yo lo he vivido ya

14 I: ¿eh? entonces / pue <alargamiento/>s estábamos en casa de mi abuela y

15 cuando<alargamiento/> llegó el ejército // que allí se había se tenía la leyenda como ahora /

(PRESEEA, MADR_M33_054)

\subsubsection{Reclamar primacía epistémica}

La segunda función se da cuando H2 realiza una elaboración de la información presentada por $\mathrm{H} 1$ como modo de constituirse como un hablante autorizado (incluso más autorizado que H1) para hablar sobre un tema. Este uso se explica fácilmente en el contexto de las entrevistas periodísticas divulgativas. Los ejemplos (9) y (10), ambos de la entrevista de Risto Mejide (RM) al filósofo y pedagogo José Antonio Marina (JAM), son ejemplos de ello. En (9), RM habla sobre los sentimientos de su madre. En (10), ambos están hablando sobre educación: RM, con formación como publicista, compara la motivación de un niño con una venta publicitaria.

En ambos casos, RM propone análisis que son corroborados y, al mismo tiempo, marcados como evidentes por JAM. Ello se realiza mediante el uso de las partículas intensificadoras 'sin duda alguna' (ejemplo 9, línea 11) y 'claro' (ejemplo 10, líneas 11 y 13) unidas a la recuperación de la causa por la cual RM estaría en lo cierto: 'porque una de las características que tiene es que se tiene que dar en un ambiente de cariño' (ejemplo 9, líneas 11-12) y 'porque lo que intentáis es dirigir el comportamiento de otra persona’ (ejemplo 10, líneas 13-14). 
Es llamativo aquí que, en los dos casos, se podría decir que las aserciones propuestas por RM están más cerca su propio territorio informacional (Goffman, 1971; Kamio, 1997). En el caso de (9), el tema que se está tratando (los sentimientos de la madre de RM), están más cerca de su propia experiencia vivencial. En el caso de (10), el tema (las intenciones de los publicistas como colectivo) están más cerca de la especialización profesional de RM, quien tiene formación como publicista. Por tanto, en este sentido, quizá cabría esperar que él fuera el hablante más autorizado (Kamio, 1997) para hablar sobre ambos temas (esto es, los sentimientos de la madre y las intenciones de los publicistas). Sin embargo, las identidades institucionales operativas en este contexto -el entrevistador y el entrevistado se comportan como aprendiz y maestro, respectivamente- posibilitan que sea JAM, en última instancia, quien tenga derecho a validar los análisis de RM.

(9)

1 RM: [esto] ¿no va en contra de esa excesiva permisividaad ee que muchas veces ahora se está

2 diciendo? ¿no? que parece que / porque a un niño le des un cachete [va]yas a acabar en el

3 calabozo [¿no?]

4 JAM: [sí] [puess] sí pues a mí per- hay quien esas cosas nos hemos pasao es decir /

$5 \quad$ castigos físicos $\mathrm{N}[\mathrm{O}]$

6 RM: [no] hombre yaa pero no es un castigo físico a mí me han dao algún

$7 \quad$ cachete al $[(())]$

8 JAM: [claro] y ¿te ha- y te eeh estás muy traumati[zao?]

9 RM: [al re]vés yo creo que le dolió más a

10 mi madre que a mís

$11 \mathrm{JAM}$ : \sin duda alguna por- porque una de las características que tiene es

12 que se tiene que dar en un ambiente de cariño

(Entrevista de Risto Mejide a José Antonio Marina en Viajando con Chester, Cuatro)

1 JAM: por eJEMplo/ ee el tema de la motivación// (PAUSA SONORA) motivar a un niño/

2 o a un adulto o a un empleao/ es/ a ver si consigo que tengas ganas de hacer/ ALgo que no

3 ten- que no tienes ganas de hacer/ pero que yo creo que debe hacer

4 RM: es una venta al final [¿ंeh?=]

5 JAM: [s-]

6 RM: es una ventaa publici[taria]

7 JAM: [sí] pero ¿quiénes- quiénes sois los expertos en motivación? 
$8 \mathrm{RM}: \quad \mathrm{hm} \mathrm{hm} /$ no no/ está claro sí sí

9 JAM: los publicIStas

$10 \mathrm{RM}$ : no no y me parece- por eso que veo que [tiene mucho=]

11 JAM: [hombre CLAro]

12 RM: =mucho en co[mún]

13 JAM: claro estuPENDdo porque lo que intentáis es $\uparrow / /$ dirigir el comportamiento $\uparrow /$ de otra

14 persona/ pero vamos a ver/ todos venimos con tres deseos (...)

(Entrevista de Risto Mejide a José Antonio Marina en Viajando con Chester, Cuatro)

Del ejemplo (10) cabe destacar la confluencia de varios elementos que parecen indicar que JAM se niega a ser informado de una determinada cuestión: es especialmente evidente, en este caso, que existe una incongruencia en términos epistémicos marcada en las diferentes contribuciones de JAM. En primer lugar, JAM corrobora el análisis de RM y, al mismo tiempo, parece oponerse mediante la conjunción adversativa 'pero' (línea 7) a algún elemento que no se puede hallar en el contenido semántico de la aserción propuesta por RM (líneas 4 y 6). No hay, en efecto, ningún elemento en el discurso de RM frente al cual el argumento de JAM en la línea 7 ('¿quiénes sois los expertos en motivación?') pueda considerarse antiorientado. Creemos que, en este caso, JAM se está oponiendo realmente a la acción misma de que se realice una aserción como si fuera desconocida por el receptor. JAM se opone, por tanto, al esquema epistémico básico del acto de habla aserción y no al contenido informativo de la aserción.

Así, se distinguen dos niveles de análisis dentro del (des)acuerdo: existe acuerdo en el plano semántico -lo propuesto por RM es cierto o válido- y desacuerdo en el plano pragmático o interaccional -el hecho de que RM proponga este contenido como novedoso es inaceptable dentro de este contexto determinado-. En esta línea apuntan también el uso de 'hombre' (línea 11) y el uso de 'vamos a ver' (línea 13). Ambas partículas discursivas, junto con 'pero', constituyen lo que podríamos considerar 'elementos formales del desacuerdo en secuencias de acuerdo' (ver García-Ramón, 2018a). En estos casos, el patrón al cual nos venimos refiriendo en este trabajo se ve reforzado por elementos que podríamos considerar marcadores de problematicidad, que señalan de forma más evidente que existe una incongruencia de algún tipo: según nuestro análisis, de naturaleza epistémica. El patrón completo podría leerse en este caso (y en casos similares) como $<$ [aserción $]_{\mathrm{H} 1}+[$ marcador de problematicidad + acuerdo + elaboración de la información $]_{\mathrm{H} 2}>$

También puede darse que uno de los hablantes se presente como el más autorizado en casos en que la asimetría entre los diferentes roles no está preestablecida por el género, sino que se co-construye sobre la marcha, en consonancia con los temas que se tratan. Es el caso de (11), de una conversación coloquial en la que dos hombres jóvenes, L y E, hablan sobre la vida sentimental de L. En este ejemplo, E interviene en 
la construcción de la descripción de una situación perteneciente a la experiencia de L. $\mathrm{El}$ análisis propuesto por $\mathrm{E}$ es corroborado por L mediante un ‘jclaro!' intensificado. Además de validar la propuesta de E, L elabora sobre esa información mediante la aportación de nuevos datos. Con este recurso, gestiona su propia primacía sobre el contenido expuesto.

1 L: pero obviamente/ ella tampoco/ que no/ [o sea]

$2 \mathrm{E}:[\mathrm{no}$ no]/ no lo dice para encabronarte/ si es

$3 \operatorname{verdad} \uparrow /$ simplemente $(())$

$4 \mathrm{~L}:$ ¡claro!/ ni y y y/ si yo le dijera/ oye mira/ no voy al cine/ ¿sabes?/ supongo que se

5 sentiría/ súper violentada/ ella también/ ¿sabes?// espero/ (RISAS)

6 E: (RISAS) $\$$

(Corpus Val.Es.Co. 2.0, Conversación 0039)

En los ejemplos (9-11), la señalización de asimetrías epistémicas sirve a los interlocutores para construir roles conversacionales específicos, bien preestablecidos por los requerimientos del molde del género (ejemplos 9 y 10), o bien construidos en momentos puntuales de la interacción (ejemplo 11) o, en otras palabras, ya sean consecuencia del contexto interactivo general (CIG) o del contexto interactivo concreto (CIC), respectivamente (Briz \& Albelda, 2013).

Asimismo, la función de señalar incongruencia epistémica se solapa en ocasiones con otra función que también tienen las partículas intensificadoras en la interacción: recuperar el turno de habla cuando este ha sido arrebatado por otro hablante. Tal es el caso de la partícula 'claro' en los ejemplos (10) y (11). Consideramos que ambas funciones (reclamar primacía epistémica y tratar de recuperar el turno) no solamente son compatibles, sino que, de hecho, se complementan y se refuerzan mutuamente. Precisamente el hecho de marcar una información como conocida u obvia crea la antesala para que $\mathrm{H} 2$ recupere el turno de palabra, sobre la base de la idea de que aquello que está diciendo $\mathrm{H} 1$ no es necesario en tanto que es excesivamente informativo en un CIC dado. Por tanto, es precisamente el señalamiento de una incongruencia lo que permite a $\mathrm{H} 2$ a constituirse de nuevo como hablante.

\subsubsection{Deslegitimar acciones conversacionales amenazantes a partir de su base epistémica}

El tercer tipo de motivación opera especialmente en casos en que H1 realiza una acción conversacional que, pese a tratarse superficialmente de una aserción, en última instancia realiza otra acción que es potencialmente amenazadora para la imagen de alguno de los interlocutores. Esta acción es deslegitimada a partir de la negación de la informatividad de la aserción propuesta por H1. Por tanto, el potencial amenazante se 
neutraliza mediante la señalización de que existe incongruencia epistémica, es decir, mediante la señalización de que la aserción propuesta por H1 tiene un contenido obvio y, por tanto, no relevante en la interacción. Tal y como afirma Grzech (2016: 377),

"Declarative clauses, even if they have directive illocutionary force, still constitute an update of the common ground, rather than an update of the to-do list (cf. Portner 2006), and as such can be reconciled with the notion of epistemic authority/primacy."

En nuestro corpus, las acciones conversacionales a las que nos referimos son críticas y autocríticas. Shaw y Hepburn (2013) proponen un análisis similar en su estudio sobre interacciones entre mujeres que acaban de ser madres primerizas y las madres de esas mujeres. En el estudio, las madres primerizas oponen resistencia a los consejos de sus madres - planteados no de forma imperativa sino como análisis de qué sería conveniente- mediante la siguiente estrategia: por una parte, las madres primerizas aceptan el contenido del consejo; por otra, rechazan la informatividad de la acción conversacional. Ello se produce porque aceptar el consejo en todas sus dimensiones equivaldría a aceptar su propio desconocimiento $\mathrm{y}$, de alguna forma, su falta de competencia en una materia importante (la crianza de su hijo).

En el corpus, el uso del patrón < acuerdo + elaboración de la información> para deslegitimar acciones conversacionales aparece en secuencias de las entrevistas políticas en que el entrevistador realiza un análisis que se podría entender como una crítica al político (ejemplos 12 y 13) y en secuencias de las entrevistas divulgativas y de las conversaciones coloquiales en que lo dicho por $\mathrm{H} 1$ puede entenderse como una autocrítica (ejemplos 14 y 15). En (12) y (13), los entrevistados -Esperanza Aguirre (EA) en (12) y Pedro Sánchez (PS) en (13) - deslegitiman una crítica dirigida a ellos mediante la señalización de que la información producida por H1 constituye una obviedad -algo que simplemente no podría ser de otra manera- y, por tanto, no puede constituir una crítica válida. En (12), EA elabora sobre la información de JE a partir de la recuperación de la causa; en (13), PS realiza una generalización del fenómeno al pasar de focalizar la discusión sobre el comportamiento de Felipe González en particular a referirse a la evolución de la sociedad en general.

$1 \mathrm{JE}$ : creo que-/ que el presupuesto de esee Consejo Consultivo de la/ Comunidad de Madrid es de

2 MÁS de CUAtro millones de euros

3 EA: bueno evi[dente=]

$4 \mathrm{JE}:\left[{ }^{\circ}\right.$ pero $\left.^{\circ}\right]$

$5 \mathrm{EA}:=$ mente es que tiene que haber letraos $\uparrow /$ tiene que haber un-

$6 \mathrm{JE}$ : pero ¿lo vee necesario?

7 EA: un edificio $\uparrow$

(Entrevista de Jordi Évole a Esperanza Aguirre en Salvados, La Sexta) 
(13)

$1 \mathrm{RM}: \dot{\mathrm{y} y}$ las puertas giratorias?

2 PS: se acabaron hay que cerrarlas

3 RM: mira Fe- Felipe González

4 PS: sí claro es que la sociedad ev- evoluCIOna/ y hoy Felipe González si DEJAra el gobierno

5 estoy convenCIDO/ estoy conven[cido=]

6 RM: [pero] ¿le=

7 PS: =que no formaría

(Entrevista de Risto Mejide a Pedro Sánchez en Viajando con Chester, Cuatro)

Mediante el mismo procedimiento, en (14) y (15), H2 deslegitima autocríticas producidas por H1. Este uso se da típicamente en entornos menos confrontacionales, como las entrevistas a expertos y las conversaciones coloquiales. En (14), de la entrevista de Jordi Évole (JE) a José Luis Sampedro (JLS), JE propone un análisis tentativo que podría entenderse como una autocrítica ('yo también debo estar en manos de los que mandan', línea 1). Ante ese análisis, JLS enfatiza la obviedad de la cuestión ('ipues claroo!') y generaliza el fenómeno para incluirse a sí mismo dentro de la posible crítica ('y yo también'). Más adelante, otra propuesta de JE ('forma parte del sistema', línea 18) vuelve a ser ratificada intensificadamente ('CLAro!', línea 20) y marcada como conocida mediante una nueva elaboración de la información en forma de generalización del fenómeno ('¡el sistema es muy listo!', línea 22). Además de deslegitimar una autocrítica producida por H1, H2 muestra que es el hablante más autorizado para hablar sobre las cuestiones tratadas y para legitimar o deslegitimar comportamientos. En (14), a pesar de que JLS es experto en economía, esta no es razón suficiente, en principio, para que pueda evaluar moralmente el comportamiento de JE. Se trata de uno de los casos en que un interlocutor, en virtud de la autoridad que le otorga el molde interaccional en sí, puede comportarse como guía moral o vital, independientemente del conocimiento técnico que posea sobre temas específicos.

$1 \mathrm{JE}: \quad \mathrm{n}-$ jyo también debo estar en manos de los que mandan!

2 JLS: ipues claroo! y yo también

3 JE: (RISAS)

4 JLS: pero yo/ me refugio en este rincóon $\uparrow /$ escribo mis pa- mis palabras que tal $\uparrow /$ y cuando me

5 invitan a venderme y vienen a comprarme/ y- exactamente igual que usted ¿verdad? / digo

6 que no

7 JE: durante los anuncios/ claro/ iGUAL se anuncian BANcos/ que en fondo ¡nos están 
8 subvencionando que nosotros podamos hacer este programa!/ y mientras les criticamos

9 JLS: claroo

$10 \mathrm{JE}$ : es super subversivo esto

11 JLS: síi bueeno pero usted eso/ eso tienee-/ eso- eso se explica también ¿verdad?/ yoo me- me

12 acuerdo eeh quee a veces/ eeh se decía/ en la universidad/ en los TIEMpos de- de- de ba/

13 quee/ iban policías a las clases/y es verdad que iban policías a las clases ee informaban $y$

$14 \mathrm{tal} / \mathrm{y}$ bueeno laas autoridades de entonces decían bueno/ que digan lo que quieran porque

15 gracias/ a que Aranguuren/ yy Tierno Galván/y Sampedro/ y te-el otro/ y unos cuantos/

16 dicen cosas/ eeh/ me-m-m-pocoo/gaLANtes con el régimen/ damos la sensación de que

17 hay libertad/ de modo que enCIMA / ¡les hacemos un favor!

$18 \mathrm{JE}$ : o sea diciendo esto / a pesar de que ahora puedan venir unos anuncioos / n- donde pueda

19 haber una entidad bancaria $\uparrow /$ bueeno/ forma parte del sistema

20 JLS: ¡CLAro! ¡forma parte del sistema!

$21 \mathrm{JE}:{ }^{\circ}$ (bueno) ${ }^{\circ}$

\section{JLS: ¡el sistema es muy listo!}

(Entrevista de Jordi Évole a José Luis Sampedro en Salvados, La Sexta)

En (15), C y P son dos mujeres que hablan coloquialmente sobre la temperatura esperable en el pueblo y la consiguiente planificación del viaje por parte de C. Ante la narración de C (líneas 1-3), que se podría entender como una autocrítica, $\mathrm{P}$ señala que no es sorprendente que $C$ no planificara mejor el viaje $y$, para concretar este uso de 'claro', recupera la causa por la cual esta mala planificación era esperable ('porque no te esperas', líneas 4-5), por lo que no es necesario que C incida más sobre esta cuestión. Todo ello se relaciona, por supuesto, con la fuerza de la instrucción de colaborar en la conversación.

$1 \mathrm{C}$ : era la Resurrección a las doce de la noche $\uparrow$ / y las chiquillas casi sin venir ((diciendo)) digo

2 mira $\uparrow$ si yo no tengo muchas ganas de ir a la Resurrección/ y es que hacía UN FRÍO que

3 pelaba y no me llevé abrigo/ me llevé ((na(da) más)) que el traje de chaqueta $\$

4 P: $₫$ ¡claro! 
5 porque no te esperas $\uparrow /$ pero noo/ cuando te vas a esos sitios [aunque NO lo $(($ uses $))=$ ]

$6 \mathrm{C:} \quad$ [pero es que $(($ te vas $\uparrow))]$

$7 \mathrm{P:} \quad=$ tienes que llevártelo $₫$

$8 \mathrm{C}: \int t o t a l \downarrow[\mathrm{el}=]$

9 P: $\quad[((y a \downarrow$ sí $\downarrow))]$

$10 \mathrm{C}:=\mathrm{el}$ domingo $\uparrow$ el [sábado=]

11 P: [si]

$12 \mathrm{C}:=$ a mediodía y dos días más $\uparrow$ tres días $\downarrow$ hija mía $\downarrow$ y llevamos el coche a tope $\downarrow$ entre lo que

13 llevaba [mi cuñada y eso]

14 P: [(( )) ¿qué quieres?] estamos en marzo/ o sea estábamos en [marzo aún] (Corpus Val.Es.Co. 2.0, Conversación 0030)

Se ha descrito el uso del patrón < acuerdo + elaboración de la información> tras acciones conversacionales que tienen una base epistémica (describen un estado de cosas o presentan un análisis determinado) y, al mismo tiempo, pueden ser interpretados como acciones conversacionales amenazantes para alguno de los interlocutores (p. ej., las autocríticas) y que, en determinados casos, pueden al mismo tiempo contener una carga directiva o impositiva importante (p. ej., críticas) ${ }^{7}$. Estas acciones difieren de otras, como las órdenes directas planteadas en modalidad imperativa, que simplemente no pueden ser analizados desde una óptica epistémica y, por tanto, no pueden ser deslegitimadas mediante la negación de su informatividad -y, en consecuencia, su deslegitimación se tendría que realizar a partir de otro tipo de proceso, no relacionado con la incongruencia epistémica-.

Resulta claro que las funciones descritas en 4.2. no son categorías discretas, sino que pueden aparecer entremezcladas en una misma secuencia. Así, por ejemplo, en (14), H2 deslegitima una autocrítica de $\mathrm{H} 1$ y se constituye como el hablante más autorizado, simultáneamente; en (15), H2 intensifica el acuerdo y, al mismo tiempo, deslegitima una autocrítica de H2; en (10), L enfatiza el hecho de que L y E están coconstruyendo un discurso y se señala a sí mismo como el hablante que, en este contexto interactivo concreto, tiene primacía epistémica sobre el referente. Asimismo, la recuperación del turno de habla es una función íntimamente ligada a la señalización de incongruencia epistémica, como se ha mostrado en el análisis (ver sección 3.2.2).

\section{CONCLUSIONES}

Hemos argumentado que, cuando aparecen insertas en el patrón <acuerdo + elaboración de la información>, las partículas modales asertivas señalan que la aserción anterior es cierta o plausible en lo que respecta a su contenido, pero problemática en términos de su configuración epistémica básica. A partir de ese 
análisis, hemos propuesto la utilidad de un análisis que parte de la distinción entre dos niveles dentro de la categoría general de (des)acuerdo. En ocasiones, la función de señalar incongruencia epistémica se emplea estratégicamente para recuperar el turno de palabra, lo cual puede indicar una cierta competitividad o ser consecuencia de la coconstrucción del discurso.

En las secuencias de acuerdo en las que se da incongruencia epistémica, se hace patente que el hablante muestra conformidad con el contenido semántico de la aserción producida por su interlocutor, al tiempo que manifiesta disconformidad con la producción de acciones conversacionales en sí y, más concretamente, con el esquema epistémico básico asociado a ese tipo de acción conversacional. En ocasiones, este tipo de disconformidad es empleada estratégicamente para deslegitimar acciones conversacionales que pueden constituir amenazas para las imágenes de los interlocutores sin necesidad de rechazar el contenido de la aserción en sí mismo. Asimismo, el análisis muestra que los roles sociofuncionales de los interlocutores están en estrecha relación con los diferentes posicionamientos epistémicos, los cuales se observan con un análisis parcial de determinados tipos de secuencias, pero que claramente tienen implicaciones en toda la interacción. Con respecto al género entrevista, se podría considerar que existe, además de un tipo de poder relacionado con los turnos de palabra y la progresión de la información, detentado por el entrevistador de forma general, otro tipo de poder de tipo epistémico, relacionado con quién es el hablante más autorizado para emitir juicios y análisis sobre determinados temas (o, como se ha visto, de forma general, sobre todos los temas).

\section{REFERENCIAS BIBLIOGRÁFICAS}

Alston, W.P. (1964). Philosophy of language. Englewood Cliffs, NJ: Prentice Hall.

Alston, W.P. (1986). Meaning. En L.E. Hahn (Ed.), The Philosophy of W. V.Quine (pp. 49-75). La Salle: Open Court.

Alston, W.P. (1991). Searle on illocutionary acts. En E. Lepore (Ed.), John Searle and his Critics (pp. 57-80). Oxford: Blackwell.

Bach, K. \& Harnish, R.M. (1979). Linguistic communication and speech acts. Cambridge, MA: MIT Press.

Barrenechea, A.M. (1979). Operadores pragmáticos de actitud oracional: Los adverbios en -mente y otros signos. En A.M. Barrenechea, M.M. de Rosetti, M.L. Freyre, E. Jiménez, T. Orecchia \& C. Wolf (Eds.), Estudios lingüisticos y dialectológicos: temas hispánicos (pp. 39-59). Buenos Aires: Hachette. 
Briz, A. (2010a). Lo coloquial y lo formal, el eje de la variedad lingǘstica. En R.M. Castañer \& V. Lagüéns Gracia (Coords.), De moneda nunca usada: Estudios dedicados a José María Enguita Utrilla (pp. 125-133). Zaragoza: Instituto Fernando el Católico.

Briz, A. (2010b). El registro como centro de la variedad situacional. Esbozo de la propuesta del grupo Val.Es.Co. sobre las variedades diafásicas. En I. Fonte Zarabozo \& L. Rodríguez Alfano (Comps.), Perspectivas dialógicas en estudios del lenguaje (pp. 21-56). México: Editorial de la Universidad Autónoma Metropolitana-Iztapalapa.

Briz, A. (2012). La constelación comunicativa coloquial. Hacia un modo más dinámico de entender lo coloquial. Español Actual, 98, 217-225.

Briz, A. (2016). Evidencialidad, significados pragmáticos y partículas discursivas en español. Sobre la intensificación tácticamente evidencial. En R. González Ruiz, D. Izquierdo Alegría \& Ó. Loureda Lamas (Eds.), La evidencialidad en español: teoría y descripción (pp. 103-128). Madrid: Iberoamericana/Vervuert.

Briz, A. \& Albelda, M. (2013). Una propuesta teórica y metodológica para el análisis de la atenuación lingüística en español y portugués. La base de un proyecto en común (ES.POR.ATENUACIÓN). Onomázein, 28, 288-319.

Briz, A. \& Grupo Val.Es.Co. (2003). Un sistema de unidades para el estudio del lenguaje coloquial. Oralia, 6, 7-61.

Cabedo, A. \& Pons, S. (en línea). Corpus Val.Es.Co. 2.0. [en línea]. Disponible en: http://www.valesco.es /?q=es/conversaciones.

Clift, R. (2006). Indexing stance: Reported speech as an interactional evidential. Journal of Sociolinguistics, 10(5), 569-95.

Clift, R. (2007). Getting there first: non-narrative reported speech in interaction. En E. Holt \& R. Clift (Eds.), Reporting Talk: Reported Speech in Interaction (pp. 120-149). Cambridge: Cambridge University Press.

Cornillie, B. \& Gras, P. (2015). On the interactional dimension of evidentials: The case of the Spanish evidential discourse markers. Discourse Studies, X, 1-21.

Couper-Kuhlen, E. \& Thompson, S. (2000). Concessive patterns in conversation. En E. Couper-Kuhlen \& B. Kortmann (Eds.), Cause, condition, concession, contrast: Cognitive and discourse perspectives (pp. 381-410). Berlin/Nueva York: Mouton/De Gruyter.

Couper-Kuhlen, E. \& Thompson, S. (2008). On assessing situations and events in conversation: 'Extraposition' and its relatives. Discourse Studies, 10, 443-467. 
Fuentes, C. (1991). Adverbios de modalidad. Verba, 18, 275-321.

Fuentes, C. (2009). Diccionario de conectores y operadores del español. Madrid: Arco/Libros.

García-Ramón, A. (2018a). Indexing epistemic incongruence: Uy as a formal sign of disagreement in agreement sequences in Spanish. Journal of Pragmatics, 131, 117.

García-Ramón, A. (2018b). Primacía epistémica en géneros interaccionales: Atenuación, intensificación y territorio. RILCE. Revista de Filología Hispánica, 34(3), 1336-64.

García-Ramón, A. (2018c). Epistemicidad en interacción: (A)simetrías epistémicas en secuencias de acuerdo y su relación con la construcción de roles funcionales en conversaciones y entrevistas. Tesis doctoral, Universitat de València, Valencia, España.

Goffman, E. (1971). The Presentation of Self in Everyday Life. Harmondsworth: Penguin.

Grzech, K. (2016). Discourse enclitics in Tena Kichwa: A corpus-based account of information structure and epistemic meaning. Tesis doctoral, SOAS University of London, Londres, Reino Unido.

Grupo Val.Es.Co. (2014). Las unidades del discurso oral. La propuesta Val.Es.Co. de segmentación de la conversación (coloquial). Estudios de Lingüística del Español, 35(1), 11-71.

Heritage, J. (2012). Epistemics in Action: Action Formation and Territories of Knowledge. Research on Langange and Social Interaction, 45(1), 1-29.

Heritage, J. (2013a). Action formation and its epistemic (and other) backgrounds. Discourse Studies, 15(5), 551-578.

Heritage, J. (2013b). Epistemics in Conversation. En T. Stivers \& J. Sidnell (Eds.), The Handbook of Conversation Analysis (pp. 370-394). Londres: Wiley-Blackwell.

Heritage, J. \& Raymond, G. (2005). The Terms of Agreement: Indexing Epistemic Authority and Subordination in Talk-in-Interaction. Social Psychology Quarterly, 68(1), 15-38.

Hutchby, I. \& Wooffitt, R. (2008). Conversation Analysis. Cambridge: Polity Press.

Kamio, A. (1997). Territory of Information. Amsterdam/Philadelphia: John Benjamins.

Maldonado, R. (2010). Claro. De objeto perceptible a refuerzo pragmático. En M.J. Rodríguez-Espiñeira (Ed.), Adjetivos en el discurso (pp. 61-107). Santiago de Compostela: Universidad de Santiago de Compostela. 
Martín Zorraquino, M.A. (1994). Sintaxis, semántica y pragmática de algunos adverbios oracionales asertivos en español actual. En V. Demonte (Ed.), Gramática del español (557-590). México D.F.: Centro de Estudios Lingüísticos y Literarios, el Colegio de México.

Martín Zorraquino, M.A. \& Portolés, J. (1999). Los marcadores del discurso. En I. Bosque \& V. Demonte (Dirs.), Gramática descriptiva de la lengua española. Vol. III (pp. 4051-4213). Madrid: Espasa Calpe.

Pomerantz, A. (1984). Agreeing and disagreeing with assessments: Some features of preferred/dispreferred turn shapes. En J.M. Atkinson \& J. Heritage (Eds.), Structures of Social Action (57-101). Cambridge: Cambridge University Press.

Pons, S. (2003). From agreeing to stressing and hedging: Spanish bueno and claro. En G. Held (Ed.), Partikeln und Höflichkeit (pp. 219-236). Bern: Peter Lang.

Pons, S. (2017). Claro. En A. Briz, S. Pons \& J. Portolés (Eds.), Diccionario de Partículas Discursivas del Español [en línea]. Disponible en: http://www.dpde.es.

Portner, P. (2006). Comments on Faller's paper. Ponencia presentada en Workshop on Philosophy and Linguistics, University of Michigan, Michigan.

PRESEEA (en línea). Corpus del Proyecto para el estudio sociolingüístico del español de España y de América. Alcalá de Henares: Universidad de Alcalá [en línea]. Disponible en: http://www.preseea.linguas.net.

Sánchez Jiménez, S.U. (2017). Naturalmente. En A. Briz, S. Pons \& J. Portolés (Eds.), Diccionario de Partículas Discursivas del Español [en línea]. Disponible en www.dpde.es.

Sacks, H. (1992). Lectures on Conversation. Londres: Blackwell.

Schegloff, J. (1992). To Searle on Conversation: A Note in Return. En H. Parret \& J. Verschueren (Eds.), (On) Searle on Conversation (pp. 113-128). Amsterdam: John Benjamins.

Searle, J. (1969). Speech Acts: An Essay on the Philosophy of Language. Cambridge: Cambridge University Press.

Searle, J. (1992). Conversation. En H. Parret \& J. Verschueren (Eds.), (On) Searle on Conversation (pp. 7-30). Amsterdam: John Benjamins.

Shaw, C. \& Hepburn, A. (2013). Managing the Moral Implications of Advice in Informal Interaction. Research on Langange and Social Interaction, 46(4), 344-362.

Sidnell, J. (2010). Conversation Analysis. An Introduction. Londres: Wiley-Blackwell. 
Sidnell, J. (2012). Who knows best? Evidentiality and epistemic asymmetry in conversation. Pragmatics and Society, 3(2), 294-320.

Sidnell, J. (2013). Basic conversation analytic methods. En T. Stivers \& J. Sidnell (Eds.), The Handbook of Conversation Analysis (pp. 77-100). Londres: WileyBlackwell.

Stivers, T. (2005). Modified Repeats: One Method for Asserting Primary Rights from Second Position. Research on Language and Social Interaction, 38(2), 131-58.

Stivers, T. (2011). Morality and question design: "of course" as contesting a presupposition of askability. En T. Stivers, L. Mondada \& J. Steensig (Eds.), The morality of knowledge in conversation (pp. 82-106). Cambridge: Cambridge University Press.

Stivers, T., Mondada, L. \& Steensig, J. (2011). Knowledge, morality and affiliation in social interaction. En T. Stivers, L. Mondada \& J. Steensig. (Eds.), The morality of knowledge in conversation (pp. 3-24). Cambridge: Cambridge University Press.

ten Have, P. (1999). Doing Conversation Analysis: A practical guide. Londres: SAGE.

Taranilla, R. (2016). La noción de patrón discursivo y su utilidad en la descripción de los marcadores del verbo parecer. En M. Borreguero Zuloaga \& S. Gómez-Jordana Ferary (Coords.), Les marqueurs du discours dans les langues romanes: une approche contrastive (pp. 233- 250). Limoges: Lambert Lucas.

Vázquez Carranza, A. (2009). Agreement and Epistemics in Mexican Spanish Talk. MA Thesis, University of Essex, Essex, Reino Unido.

Vázquez Carranza, A. (2016). Aceptación y resistencia: un análisis de 'ah' y 'ay' como indicadores de cambio de estado. Cuadernos de Lingüistica de El Colegio de México, 3(2), 71-103.

Vázquez Carranza, A. (2017). Some uses of 'no' in Spanish talk-in-interactions. International Review of Pragmatics, 9, 1-24.

\section{NOTAS}

${ }^{1}$ Este trabajo se ha llevado a cabo en el marco del proyecto de investigación Es.Vag.Atenuación: La atenuación pragmática en su variación genérica: géneros discursivos escritos y orales en el español de España y América (MINECO: FFI2016-75249-P) y ha contado con una ayuda para la Formación del Profesorado Universitario (MECD: FPU13/03399).

2 Empleamos en este trabajo la noción de 'acto de habla' porque es que generalmente se emplea en los estudios de Pragmática. Sobre la problemática en torno a la aplicación de la 
noción de 'acto de habla' al análisis de interacciones reales, de la cual no nos ocupamos en este trabajo, ver, entre otros, las propuestas de Alston $(1964,1986,1991)$ y Bach y Harnish (1979) o el debate entre Schegloff y Searle (Schegloff, 1992; Searle, 1992).

${ }^{3} \mathrm{Si}$ bien es cierto que se tiende a identificar el uso de estas partículas con el mayor grado de seguridad (Barrenechea, 1979), para algunos autores el grado máximo de seguridad es en realidad 'la aseveración en sî́: esta perspectiva alternativa se basa en la idea de que las partículas modales asertivas "niegan una posible presuposición de duda" (Fuentes, 1991: 310) y, por tanto, se reconoce mediante ellas que podría existir tal duda. No nos ocuparemos en este trabajo de esta problemática.

${ }^{4}$ Es posible, potencialmente, describir estructuras que señalen otro tipo de incongruencias (p. ej., deónticas). Sin embargo, puesto que todas las acciones conversacionales analizadas en este trabajo tienen base epistémica, puesto que son siempre asertivas, la incongruencia recibe aquí siempre ese apellido.

5 Pons (2003) (ver también Pons (2017)) analiza el patrón 'claro + porque' para reforzar un argumento co-orientado con el presentado por el otro hablante. Estos 'movimientos justificativos' descritos por el autor se incluirían dentro de lo que aquí llamamos 'señalamiento de la causa'.

${ }^{6}$ Esta idea se desarrollará a lo largo del análisis. Sin embargo, sirva como ilustración preliminar una situación hipotética en que un hablante (H1) se justifica ante otro (H2) por un error cometido. En este caso, si H2 señala que existe incongruencia epistémica (es decir, si H2 expresa que la justificación es en gran medida redundante) está llevando a cabo, en realidad, un movimiento conversacional afiliativo mediante el cual refuerza los lazos con $\mathrm{H} 1$.

${ }^{7}$ La carga directiva es quizá más evidente en los consejos descritos por Shaw y Hepburn (2013), pero no deja de estar presente, aunque sea de forma más velada, en las críticas a un político por parte de un entrevistador que, en una entrevista política, tiene funciones de árbitro o de evaluador y que, en última instancia, tiene la capacidad de pedir explicaciones. 